\title{
Alveolar and symphysis regions of patients with skeletal class II division 1 anomalies with different vertical growth patterns
}

Elcin Esenlik'

Fidan Alakus Sabuncuoglu ${ }^{2}$

\section{ABSTRACT}

Objectives: The aim of this study was to investigate the alveolar and symphysis region properties in hyper-, hypo-, and normodivergent Class II division 1 anomalies.

Methods: Pretreatment lateral cephalograms of 111 young adult female patients with skeletal Class II division 1 anomalies were compared to those of 54 Class I normal subjects (control group). Class II cases were divided into hyperdivergent ( $n=58$ ), hypodivergent ( $n=19$ ), and normodivergent groups ( $n=34$ ). The heights and widths of the symphysis and alveolus and the depth of maxillary palate were measured on the lateral cephalograms.

Results: Mean symphysis width was wider in the hypodivergent Class II group than in the other groups, while mean symphysis height was similar among all groups. Maxillary palatal depth, upper incisor angle, upper and lower molar alveolar heights, and Id-ld' width were also similar among groups.

Conclusion: Symphysis width is the main factor in the differential diagnosis of Class II division 1 anomaly rather than symphysis height and hypodivergent Class II Division 1 anomaly is more suitable for mandibular incisors movements. (Eur J Dent 2012;6:123-132)

Key words: Dentoalveolar structure; symphysis region; class II division 1 anomaly; vertical pattern

1 Department of Orthodontics Faculty of Dentistry, Suleyman Demirel University, Isparta, TURKIYE

2 Erzurum Maresal Cakmak Military Hospital Dental Center, Department of Orthodontics, Erzurum, TURKIYE

- Corresponding author: Dr. Elcin Esenlik Suleyman Demirel Universitesi, Dishekimligi Fakultesi, Ortodonti A.D., 32260 Cunur, Isparta, TURKIYE

Tel: +902462118807

Fax: +902462370607

Email: elcinesवyahoo.com

\section{INTRODUCTION}

The characteristic properties of skeletal Class II division 1 anomalies have been investigated by several authors in terms of angle classification. A wide variety of skeletal types are thought to be found in the larger population designated as Class II'. Several authors have divided Class II anomalies into subgroups. ${ }^{1-5}$ Henry $^{2}$ stated that Class II division 1 malocclusions vary considerably and may be 
classified into 4 types: maxillary protrusion, maxillary basal protrusion, micro-mandible, and basic mandibular retrusion. He also stated that because of the superimposition of environmental factors on hereditary influences, some Class II cases involve a combination of these 4 types. Steep mandibular and occlusal planes with apparent forward slopes of the lower incisors are typical of the micro-mandible type. Sassouni ${ }^{5}$ also assessed Class II division 1 cases according to vertical type and subdivided them into 4 groups: 2 types of Class II deep bites, 1 with mandibular retrusion and 1 with maxillary protrusion; and 2 types of Class II open bites, 1 with mandibular retrusion and 1 with maxillary protrusion. He emphasized that the divergent treatment plan was primarily for the vertically unbalanced. He also stressed that differential diagnosis of each Class II division 1 case is critical.

Facial types of a multidimensional nature are derived from the combination of anteroposterior and vertical dimensions. Teeth, muscles, and bones interact intimately during growth, increasing or masking initial deformities. ${ }^{5}$ Disproportions and malpositions of the structures often lead to malocclusions or facial deformities.

Hellman ${ }^{6}$ found that the Class II division 1 mandibular angle was more acute than the mandibular angle of skulls exhibiting normal occlusion. Similarly, Drelich ${ }^{7}$ stated that a relatively steeper mandibular plane (MP) angle existed in Class II division 1 cases. Schudy 4 stated that use of the MP angle is useful in describing different facial types and should be taken into consideration in treatment planning. Bishara and Augspurger ${ }^{8}$ reported that changes in the MP angle are associated with facial and dental characteristics. Thus, use of this angle could assist clinicians in identifying facial types that are the result of the cumulative effect of various genetic and environmental factors during an individual's development. They also reported that a single parameter such as MP angle should not be expected to accurately reflect all of these interactions.

In orthodontics, knowledge of mandibular growth is highly beneficial in diagnosis and treatment planning and is critical in the development of balanced dentofacial structures ${ }^{9}$. Mandibular rotation types have been well defined by several authors. ${ }^{10,11}$ It was stated that some additional growth at the major growth sites accompanied by mandibular rotation and remodeling tend to reshape the mandible. These remodeling changes described for the inferior mandibular border have been related to rotational changes in the mandible. ${ }^{12}$ Anterior rotation is generally associated with deposition in the inferior aspect while posterior rotation is associated with resorption. ${ }^{13}$ These remodeling processes were thought to be indirectly related to the nature of the stresses generated by the supra-hyoid musculature. However, the manifestation mechanism is perhaps more complex than expected.

It is believed by some authors that the symphysis region properties could be a good indicator of mandibular rotation. ${ }^{9,10,14,15}$ The symphysis is one of the most important regions of the craniofacial complex for clinical orthodontists, and it serves as a primary reference for esthetic considerations in the lower one-third of the face. ${ }^{12}$ Furthermore, the vertical and sagittal positions of the mandibular incisors are important determinants in planning occlusal and skeletal relations for orthodontic treatment and orthognathic surgical procedures. Therefore, investigation of the structure of the alveolar bone is essential for differential diagnosis.

Dentoalveolar structures are the most intervened by orthodontists, and are the site for most pathological conditions encountered during treatment. Therefore, it remains a current issue in orthodontics. Biological and biomechanical factors are closely related to the potential side effects of orthodontic treatment, including external root resorption, bony dehiscence and fenestration, and gingival retraction. ${ }^{16}$ Limited movement of the incisors during orthodontic treatment depends on the involved alveolar bone structure. It is important to determine the incisor movement limitations for orthognathic surgery or camouflage treatments of Class II division 1 cases to maintain stabilization and avoid iatrogenic sequelae. Especially in borderline cases, these structures should be considered in detail while considering treatment options and to minimize iatrogenic factors. Although several investigations have examined different vertical types of Class II patients, few studies have focused on the alveolar structures of these types of Class II patients. ${ }^{6,17,18}$ Therefore, the aim of this study was to investigate the differences in the properties of the alveolar and symphysis regions between hyperdivergent, hypodivergent, and nor- 
modivergent skeletal Class II division 1 anomalies and a Class I control group.

\section{MATERIALS AND METHODS}

In this study, patients with skeletal Class II division 1 anomalies referred to orthodontics clinic between 2007-2010 years were evaluated and pretreatment lateral cephalograms of 163 adult female patients were retrieved from the archives of the Department of Orthodontics. Patients with congenital anomalies and hypodontia were excluded from the study. A total of 21 patients exhibiting normal profiles were excluded and 31 patients having an ANB angle in the normal range were also excluded. Therefore, the study material consisted of the pretreatment lateral cephalograms of 111 skeletal Class II division 1 young adult female patients with mandibular retrognathy and a convex profile (mean age: 16,78 $\pm 2,4$ year). Patients were divided into hyperdivergent (GoGnSN> $35^{\circ} ; n=58$ ), hypodivergent ( $G o G n S N<29^{\circ} ; n=19$ ), and normodivergent (GoGnSN, 29-35०; $n=34$ ) groups. These
Class II groups were compared to an untreated Class I control group ( $n=54$; mean age:17,4 $\pm 2,9$ year). The control group exhibited normal ANB angles, normal overjet, and acceptable occlusion. All cephalograms were traced manually by a single examiner using a protractor with $0.5^{\circ}$ and $0.5 \mathrm{~mm}$ accuracy. In addition to SNA, SNB, ANB angles, Wits appraisal, MP angle (GoGnSN), and gonial angles (ArGoMe and SGoMe), symphysis width and height, upper (1/PP) and lower (1/MP) incisor angle, maxillary palatal depth, mandibular and maxillary incisors, and molar alveolar heights were measured on the lateral cephalograms. The width of the thinnest region of the symphysis at the level of the $B$ point $\left(B-B^{\prime}\right)$ and the width of the cervical region of the lower central incisor at the cemento-enamel junction (Id-Id') were evaluated. All parameters were defined as follows, and the dentoalveolar and symphysis parameters are shown in Figure 1. Measurement reliability was controlled by the examiner retracing all of the radiographs after 1 month.

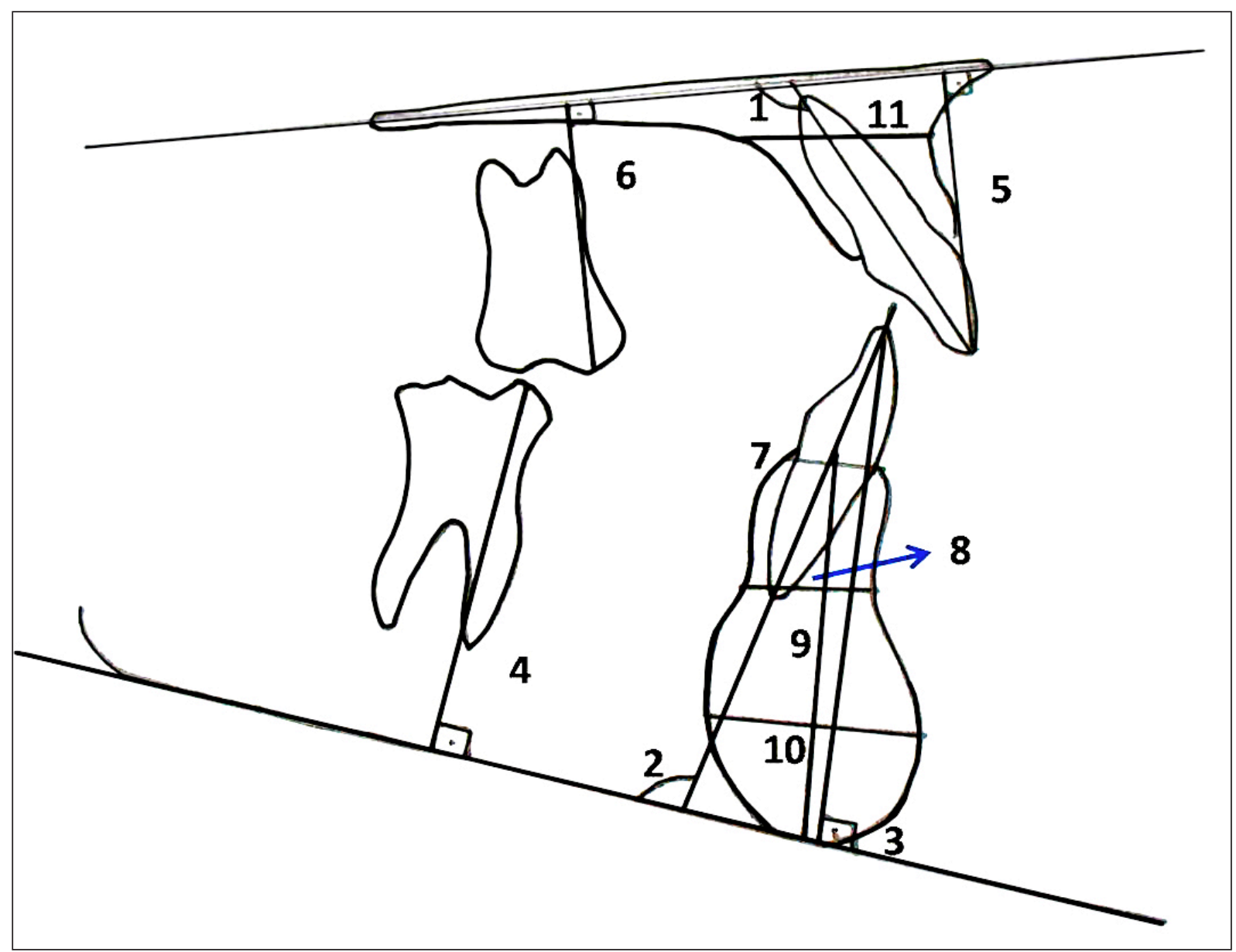

Figure 1. Dentoalveolar and Symphysis Parameters. 1: 1/PP, 2: 1/MP, 3:L1/AH, 4:L6/AH, 5:U1/AH, 6:U6/AH, 7:Id-Id' width, 8:B-B' width, 9: Symphysis height, 10: Symphysis width, 11: maxillary depth.

April 2012 - Vol.6 
Dentoalveolar and Symphysis Parameters

1-1/PP $\left({ }^{\circ}\right)$ : The angle from the maxillary central incisor's axis to the palatinal plane (ANS-PNS).

$2-1 / M P\left({ }^{\circ}\right):$ The angle from the mandibular central incisor's axis to the mandibular plane (GoMe).

3-Mandibular incisor dentoalveolar height (L1$\mathrm{AH}$ ): The perpendicular distance between the lower incisor tip and mandibular plane.

4-Mandibular molar dentoalveolar height (L6-AH): The perpendicular distance between the lower first molar mesial cusp tip and mandibular plane.

5-Maxillary incisor dentoalveolar height IU1$\mathrm{AH}$ ): The perpendicular distance from the upper incisor tip to the palatal plane.

6-Maxillary molar dentoalveolar height IU6$\mathrm{AH}$ ): The perpendicular distance from the upper first molar mesial cusp tip to the palatal plane.

7-Id-Id' width: The distance between the most anterosuperior (Id) and most posterosuperior (Id') points on the mandibular alveolus.

8-B-B' width: The distance between the $B$ and $B^{\prime}$ (the lingual projection of the $B$ point at the lingual symphysis borderl points.

9-Symphysis height: The distance from the midpoint of the anterior alveolus to the Menton point. The symphysis ratio was calculated by dividing the symphysis height by the symphysis width.

10-Symphysis width: The perpendicular distance from the pogonion to the most convex point of the lingual curvature of the symphysis.

11-Maxillary depth: The distance from the deepest point on the palatal bone curvature to the A point.

\section{Statistical Analysis}

Statistical analyses and calculations were performed using SPSS for Windows version 15.00 (SPSS Inc., Chicago, IL, USA) and Excel 2003 (Microsoft Corporation, Redmond, WA, USA). The reliabilities of the parameters were examined using intra-class correlation coefficients and were in the range $86-99 \%$. Descriptive statistics are expressed as medians, (IQR) due to the unequal sample sizes. Correlations were assessed using the Spearman's rank correlation method. Groups were compared using the Kruskal-Wallis nonparametric analysis of variance test followed by the Mann-Whitney test with Bonferroni correction for intergroup compari- sons if an overall level of significance of $P \leqslant .05$ was reached.

Sample Size and Power: A priori sample size was calculated with $G^{*}$ Power (G*Power Ver. 3.0.10, Franz Faul, Universität Kiel, Germany). For a power of $95 \%$ with an $\mathrm{f}=.30$ effect size, $\mathrm{a}=.05$ Type $\mathrm{I}$, and $B=.05$ Type II error rates, at least 49 in each group and a total of 196 patients were evaluated. Power of $>80 \%$ could be obtained with the actual number of samples for the study.

\section{RESULTS}

Median and IQR values of the angular and linear measurements and differences among the groups are given in Table 1. Post hoc pairwise comparisons for the parameters that are statistically significant among the groups are shown in Table 2.

GoGnSN, ArGoMe, and SGoMe angles were significantly different between the control and hyperdivergent and the control and hypodivergent groups as expected $(P<.001)$. These angles were similar in the control and normodivergent groups.

The 1/PP and 1/MP angles were higher in all Class II groups than in the control group, but only the 1/MP angle was significantly different $(P<.05)$. The U1/AH and LI/AH measurements in the Class II hyperdivergent group were significantly greater than those of the hyperdivergent and control groups $(\mathrm{P}<$.05). No statistically significant differences were found in the U6/AH and $\mathrm{L} 6 / \mathrm{AH}$ measurements among the groups. The Id-Id' width and B-B' width measurements were similar among the groups.

Symphysis height was greater in the hyperdivergent group than in the control and hyperdivergent groups $(1.5 \mathrm{~mm})$ but was not statistically significant. Symphysis width was higher in the hypodivergent group than in the other groups $(P<.05)$ but there was no difference among the control, hyperdivergent, and normodivergent groups. The symphysis ratio was significantly lower in the hypodivergent group than the hyperdivergent and normodivergent groups $(P<.01)$. The maxillary depth was greater in control group than all Class II groups but was not statistically significant.

\section{Correlations}

The correlation between GoGnSN angle and IdId' width in all groups, and GoGnSN angle and symphysis width in all Class II groups were not statisti- 
cally significant. GoGnSN was negatively correlated with symphysis width and positively correlated with symphysis height in only the control group $(P<.001$ and $P<.01$, respectively). This angle was also significantly negatively correlated with $B-B^{\prime}$ width in the control and hyperdivergent groups $(P<.01)$ but not in the hypodivergent and normodivergent groups.

No correlation was found between ArGoMe angle and symphysis width, height, Id-Id' width, or B-B' width in the hyperdivergent and normodivergent groups. ArGoMe angle showed a weak negative correlation with symphysis height, symphysis width, and Id-Id' width $(P<.05)$ in the control group. ArGoMe was also moderately and negatively correlated with symphysis height and $B-B^{\prime}$ width $(P<.05)$.

SGoMe angle was not found to be correlated with Id-Id' width in any of the groups but was found to be poorly and negatively correlated with B-B' width and moderately and negatively with symphysis height in only the hypodivergent group $(P<.05)$. This angle was poorly and negatively correlated with symphysis width in the control group only $(P<.05)$.

1/PP was correlated with maxillary depth (poorly and negatively) in only the normodivergent groups $(\mathrm{P}<.05)$. 1/MP showed no correlation with symphysis width in any of the groups, nor did it show any correlation with symphysis height in the control and hyperdivergent groups, whereas it was moderately and positively correlated with the hypoand normodivergent groups $(\mathrm{P}<.05$ and $\mathrm{P}<.01$, respectively).

\section{DISCUSSION}

The differential evaluation of facial types is important, as the prognosis and treatment approaches may differ by diagnosis. ${ }^{5}$ Karlsen ${ }^{15}$ reported that high and low angle cases exhibit completely different craniofacial growth patterns between 6-12 years of age. Isaacson et $\mathrm{al}^{11}$ concluded that facial growth relative to a cranial baseline proceeds along a vector composed of variable amounts of horizontal forward growth and vertical downward growth. Relative proportions of forward and downward growth have been characterized as relatively individual vectorial directions of facial growth.

It has been reported that most Class II patients exhibit vertical dysplasia. ${ }^{20-22}$ Class II patients with normal vertical patterns and with hypodivergent patterns followed this group, respectively. However, Altemus ${ }^{22}$ reported that although the Class II division 1 group has more vertical dysplasia than

Table 1. Median and interquartile range values of the parameters and comparison of differences among groups.

\begin{tabular}{|c|c|c|c|c|c|c|c|c|c|c|}
\hline \multirow[t]{2}{*}{ Parameters } & \multicolumn{2}{|c|}{$\begin{array}{l}\text { Control } \\
(n=54)\end{array}$} & \multicolumn{2}{|c|}{$\begin{array}{l}\text { Cl II-hyperdiv. } \\
\quad(n=58)\end{array}$} & \multicolumn{2}{|c|}{$\begin{array}{l}\text { Cl II-Hypodiv. } \\
\qquad n=19)\end{array}$} & \multicolumn{2}{|c|}{$\begin{array}{l}\text { Cl II-Normodiv. } \\
\text { (n=34) }\end{array}$} & \multicolumn{2}{|c|}{ Kruskal-Wallis } \\
\hline & median & IQR & median & IQR & median & IQR & median & IQR & $c^{2}$ & $\mathrm{p}$ \\
\hline SNA $\left.1^{\circ}\right)$ & 80,0 & 5,0 & 79,0 & 3,0 & 82,0 & 2,3 & 81,0 & 4,0 & 20,481 & $<0,001$ \\
\hline SNB $\left({ }^{\circ}\right)$ & 78,0 & 5,3 & 72,0 & 4,3 & 76,0 & 2,3 & 74,5 & 3,0 & 27,771 & $<0,001$ \\
\hline ANB $\left({ }^{\circ}\right)$ & 2,0 & 2,0 & 7,0 & 2,0 & 6,0 & 2,3 & 7,0 & 3,0 & 3,383 & $<0,001$ \\
\hline Wits & $-1,0$ & 3,5 & 6,0 & 5,1 & 5,0 & 3,6 & 6,8 & 4,6 & 1,956 & $<0,001$ \\
\hline Go-Gn-SN() & 31,0 & 7,3 & 40,0 & 6,0 & 26,5 & 3,0 & 33,0 & 3,3 & 90,481 & $<0,001$ \\
\hline ArGoMe $\left({ }^{\circ}\right)$ & 125,0 & 7,1 & 128,5 & 7,3 & 119,5 & 7,8 & 122,0 & 6,3 & 39,482 & $<0,001$ \\
\hline SGoMe $\left({ }^{\circ}\right)$ & 110,5 & 8,9 & 115,0 & 9,0 & 103,0 & 6,3 & 108,5 & 6,3 & 46,834 & $<0,001$ \\
\hline $1 / \mathrm{PP}\left({ }^{\circ}\right)$ & 111,0 & 9,0 & 114,0 & 9,0 & 114,5 & 9,5 & 114,5 & 14,5 & 0,287 & 0,866 \\
\hline $1 / \mathrm{MP}\left({ }^{\circ}\right)$ & 90,5 & 6,3 & 98,0 & 8,0 & 105,0 & 14,3 & 99,5 & 8,3 & 6,266 & 0,044 \\
\hline L1-AH (mm) & 40,8 & 3,0 & 42,8 & 4,0 & 40,8 & 6,0 & 42,0 & 4,1 & 6,068 & 0,048 \\
\hline L6- AH (mm) & 31,5 & 2,5 & 31,0 & 3,0 & 32,0 & 3,5 & 30,5 & 3,1 & 1,771 & 0,412 \\
\hline U1-AH (mm) & 29,8 & 4,0 & 31,3 & 3,3 & 28,8 & 3,3 & 31,0 & 4,6 & 8,464 & 0,015 \\
\hline U6- $\mathrm{AH}(\mathrm{mm})$ & 23,8 & 3,1 & 25,3 & 3,1 & 23,8 & 4,9 & 26,0 & 4,0 & 3,449 & 0,178 \\
\hline İd-id' width & 7,0 & 1,0 & 7,0 & 1,0 & 6,8 & .5 & 6,5 & 1,0 & 1,750 & 0,417 \\
\hline B-B' width (mm) & 7,0 & 1,0 & 8,0 & 3,0 & 9,8 & 2,6 & 8,0 & 3,0 & 5,604 & 0,061 \\
\hline Sym-Height (mm) & 32,5 & 2,5 & 34,0 & 4,0 & 32,3 & 5,5 & 33,0 & 2,9 & 3,893 & 0,143 \\
\hline Sym-width (mm) & 15,0 & 2,0 & 15,0 & 2,8 & 17,0 & 4,1 & 15,5 & 3,0 & 6,881 & 0,032 \\
\hline Sym h/w & 2,1 & 0,3 & 2,3 & 0,5 & 1,9 & 0,6 & 2,1 & 0,4 & 12,511 & 0,002 \\
\hline Maxilla depth (mm) & 17,5 & 3,5 & 14,0 & 4,0 & 14,5 & 4,3 & 15,0 & 3,0 & 3,309 & 0,191 \\
\hline
\end{tabular}


the normal subjects in his study, the normal group may have a vertical dysplasia or the Class II division 1 group may have no vertical dysplasia.

In addition to MP angle, gonial and lower gonial angles are used as indicators for case treatment plans and prognosis. Hellman ${ }^{6}$, working on skulls, concluded that Class II division 1 malocclusion exhibited a more acute gonial angle than skulls with excellent occlusion. He postulated that this would account for the mandible being in a more distal than the maxilla. In contrast, Gilmore ${ }^{23}$ reported that no statistically significant differences were found in the gonial angle between the adult Class II division 1 cases and normal cases. Similarly, Blair $^{24}$ also reported that comparison of Class I and Class II division 1 means in females revealed no significant differences in any of the linear or angular measurements. However, the author evaluated Class II groups according to Angle's classification.

Significant negative correlations were found in the present study between the GoGnSN angle and B-B' width in the hyperdivergent Class II and control groups. Gonial and lower gonial angles also showed a negative correlation with B-B' width in only the hypodivergent group. Gonial and GoGnSN angles also showed a negative correlation with symphysis width in only the control group. Id-ld' width was not correlated with GoGnSN or gonial angles in any group except for a negative correla-

Table 2. Post hoc pairwise comparisons between groups.

\begin{tabular}{|c|c|c|c|c|}
\hline & Group & $\begin{array}{c}\text { Hyperdiv. } \\
\text { Z (p) }\end{array}$ & $\begin{array}{c}\text { Hypodiv. } \\
\text { Z (p) }\end{array}$ & $\begin{array}{c}\text { Normodiv. } \\
\text { Z (p) }\end{array}$ \\
\hline & Control & 0.594 (0.553) & $3.281(0.001) *$ & $1.634(0.102)$ \\
\hline \multirow[t]{3}{*}{ SNA $\left.1^{\circ}\right)$} & Hyperdiv Cl II & & $4.121(<0.001) *$ & $2.670(0.008)$ * \\
\hline & Hypodiv CIII & & & $2.431(0.015)^{*}$ \\
\hline & Control & $6.612(<0.001) *$ & $1.931(0.053)$ & $4.280(<0.001) *$ \\
\hline \multirow[t]{3}{*}{ SNB $\left({ }^{\circ}\right)$} & Hyperdiv Cl II & & $4.609(<0.001) *$ & $3.441(0.001) *$ \\
\hline & Hypodiv Cl II & & & $2.658(0.008) *$ \\
\hline & Control & $7.090(<0.001) *$ & $4.133(<0.001) *$ & $1.812(0.069)$ \\
\hline \multirow[t]{3}{*}{ GoGnSN $\left({ }^{\circ}\right)$} & Hyperdiv Cl II & & $6.525(<0.001) *$ & $7.961(<0.001) *$ \\
\hline & Hypodiv Cl II & & & $6.021(<0.001) *$ \\
\hline & Control & $2.992(0.003) *$ & $4.061(<0.001) *$ & $2.308(0.021)$ \\
\hline \multirow[t]{3}{*}{$\operatorname{ArGoMe}\left({ }^{\circ}\right)$} & Hyperdiv Cl II & & $5.442(<0.001) *$ & $4.468(<0.001) *$ \\
\hline & Hypodiv Cl II & & & $2.231(0.026)$ \\
\hline & Control & $3.111(0.002) *$ & $4.607(<0.001) *$ & $1.832(0.067)$ \\
\hline \multirow[t]{3}{*}{ SGoMe $\left({ }^{\circ}\right)$} & Hyperdiv Cl II & & $5.814(<0.001) *$ & $4.640(<0.001) *$ \\
\hline & Hypodiv CI II & & & $3.579(<0.001) *$ \\
\hline & Control & $5.693(<0.001) *$ & $4.947(<0.001) *$ & $5.614(<0.001) *$ \\
\hline \multirow[t]{3}{*}{$1 / \mathrm{MP}\left({ }^{\circ}\right)$} & Hyperdiv Cl II & & $2.466(0.014)^{*}$ & $1.041(0.298)$ \\
\hline & Hypodiv Cl II & & & $1.532(0.126)$ \\
\hline & Control & $3.029(0.002) *$ & $1.058(0.290)$ & $1.461(0.144)$ \\
\hline \multirow[t]{3}{*}{ U1-AH (mm) } & Hyperdiv Cl II & & $2.992(0.003) *$ & $0.921(0.357)$ \\
\hline & Hypodiv Cl II & & & 1.816 (0.069) \\
\hline & Control & $3.961(<0.001) *$ & 0.095 (0.925) & $1.788(0.074)$ \\
\hline \multirow[t]{3}{*}{ L1-AH (mm) } & Hyperdiv Cl II & & $2.347(0.019)$ & $1.477(0.140)$ \\
\hline & Hypodiv Cl II & & & 0.939 (0.348) \\
\hline & Control & $0.038(0.970)$ & $2.545(0.011)^{*}$ & $1.353(0.176)$ \\
\hline \multirow[t]{3}{*}{ Sym-width (mm) } & Hyperdiv Cl II & & $2.566(0.010)^{*}$ & $1.050(0.294)$ \\
\hline & Hypodiv Cl II & & & $1.697(0.090)$ \\
\hline & Control & 1.727 (0.084) & $2.553(0.011)^{*}$ & 0.026 (0.979) \\
\hline \multirow[t]{2}{*}{ Sym-h/w } & Hyperdiv Cl II & & $3.404(0.001) *$ & $1.267(0.205)$ \\
\hline & Hypodiv Cl II & & & $2.553(0.011)^{*}$ \\
\hline
\end{tabular}

* Statistically significancy after Bonferroni corrections 
tion between ArGoMe and Id-Id' width in the control group. Interestingly, Id-Id' width did not exhibit any special characteristics, but the lower incisor angle (1/MP) increased significantly in all Class II groups as seen in the study by Kim et al. ${ }^{18}$ Similarly, the upper incisor angle (1/PP) showed a correlation with maxillary depth only in the normodivergent group.

Craig $^{3}$ stated that the angle of the upper incisor to the palatal plane is significantly greater and that the angle of the upper first incisor to the lower incisor is significantly smaller in Class II division 1 samples, reflecting the labial tipping of the upper and lower incisors. Buschang et $\mathrm{al}^{12}$ also stated that there were significant correlations between the inclination of the upper and lower incisors and the MP angle and between the mandibular incisor position and symphyseal remodeling. They also stated that the position of the dentition is subject to a number of epigenetic factors such as oral habits that affect both dentition and alveolar structures.

Although maxillary incisor angles were slightly increased $\left(3.5^{\circ}\right)$ and maxillary depths were decreased in all Class II types, these values were not statistically significant in the present study. When considering correlations between the 1/PPo and maxillary depth, there were none in the Class II groups but a negative correlation in the Class I group.

Tweed $^{19}$ indicated that the inclination of the lower incisors varies with the cant of the MP. The normal value of the lower incisor angle was stated in the literature as being $90^{\circ}$ with a range of $5^{\circ}$ $19,22,25,26$. In the present study, the inclination of the lower incisors increased (maximum increase was seen in the hypodivergent groupl to a statistically significant extent in all types of Class II division 1 cases as compared to the control group (Table 1). In contrast to the study by Kim et al, ${ }^{18} 1 / \mathrm{MP}$ angle also increased in the hyperdivergent group. The increased mandibular incisor angles probably resulted from the dentoalveolar compensation related to skeletal Class II. But it remains unclear whether the amount and direction of the dentoalveolar compensating mechanism occurs in different Class II types.

Maxillary alveolar processes and the mandibular condyle and alveolar processes were defined as the one of the major sites of bony additions. ${ }^{15}$ In Class II hyperdivergent cases, downward and backward rotation of the mandible is associated with excessive extrusion of the molars. ${ }^{5}$ Isaacson ${ }^{11}$ stated that the maxillary posterior alveolar process was found to be much more important than the posterior mandibular alveolar process in contributing to vertical development. In contrast to those of earlier studies, ${ }^{27,28}$ maxillary and mandibular molar heights in the present study were found to be similar among all groups, although the mean mandibular molar height was increased slightly in the hyperdivergent cases as compared to those of the other groups.

Karlsen ${ }^{15}$ stated that alveolar process growth demonstrated compliance with mandibular rotation. He also stated that dentoalveolar mechanisms have great potential for compensating for vertical skeletal deviations. Overdevelopment of the anterior lower facial height was compensated by marked growth of the incisal heights in both jaws. In his study evaluating low and high angles in patients 6-12 years of age, he found great increases in the upper and lower incisor heights in the high angle cases. As the mandible is displaced downward and forward, supraeruption of the incisors fills the created space. ${ }^{12}$ Mandibular rotation also interacts with vertical growth, and anterior or counterclockwise rotation might be expected to limit vertical change in the anterior region. Baumrind et $\mathrm{al}^{29}$ investigated the amount of modeling at the apices of the mandibular and maxillary incisors and molars associated with remodeling changes in the mandible from 8.5 to 15.5 years of age. They observed statistically significant tooth displacements associated with surface remodeling in the maxilla at all timepoints, whereas significant differences in the mandible were observed only in the vertical direction in the incisors. Janson et a ${ }^{30}$ stated that the lower anterior dental height was significantly different in each facial type. When we compared anterior alveolar heights in the present study, we found that the upper and lower incisor heights were increased slightly but significantly in the hyperdivergent group as seen in Karlsen's ${ }^{15}$ study. A severe openbite was not encountered in the hyperdivergent cases. This finding was suggestive of existing dentoalveolar compensation in hyperdivergent patients as well.

Buschang et $\mathrm{al}^{12}$ reported that boys and girls displayed considerably different changes, especially in the upper part of the symphysis during pu- 
berty. In fact, it may be difficult to identify the same structures over time because of the extent of relocation. Rosenstein31 found that the anteroposterior dimension of the symphyseal outline increased to $3.66 \mathrm{~mm}$ in boys and $1.93 \mathrm{~mm}$ in girls 8-17 years of age. He stated that this measurement was always higher in boys at any given age and that the highest increase in girls appeared at 12-13 years of age but was negligible thereafter. The amount of apposition seen at the pogonion suggests factors of molding and strengthening rather than factors mainly related to size increases. Furthermore, Garn et al, ${ }^{32}$ in a study of $>400$ subjects covering 2 full generations, stated that both symphyseal height and symphyseal thickness show evidence of genetic control. Sex differences in the symphyseal region were also reported in another study. ${ }^{12}$ Therefore, late adolescent and young adult female patients were evaluated in the present study.

Many clinicians classify the growth pattern of the mandible anteriorly or posteriorly according to the symphysis shape and size. ${ }^{15}$ Symphysis ratio in particular was found to be strongly related to the direction of mandibular growth ${ }^{9}$. Noh et al $^{33}$ found that a high symphysis ratio presented high correlations with the hyperdivergent pattern and increased gonial angle as seen in the present study. In contrast, Kim and Son ${ }^{34}$ found no statistically significant difference in symphysis ratio to mandibular plane between forward and backward rotational growth patterns. Regarding rotational growth patterns of the mandible and mandibular symphysis, anterior alveolar and skeletal relationships were correlated with IMPA and symphysis width as seen in the present study.

It was stated that the influence of an abnormal vertical skeletal pattern on symphyseal morphological characteristics is greater than that of an abnormal sagittal skeletal pattern. ${ }^{35}$ Morphological differences in the symphyseal region between Class II and Class I malocclusions were found in the present study. Gracco et $\mathrm{al}^{36}$ investigated whether any correlations exist between the morphology of the mandibular symphysis and the various facial types via volumetric computed tomography (CT) and found that the total thickness of the symphysis was greater in short-faced subjects than in long-faced subjects. In the present study, although no statistically significant differences in B-B' width among the different facial types were found, this measurement was greater in hypodivergent cases than in the other groups. Another finding of the Gracco et $\mathrm{al}^{36}$ study was that the vestibular portion of the cancellous bone thickness of the symphysis is greater at the central incisors in short-faced subjects than in long-faced subjects. These findings indicate that the greater symphysis width and B-B' width providing a wider range of incisor movement and fewer incisor protrusion movements in camouflage treatment of Class II division 1 cases may be preferred in hypodivergent Class II cases rather than in hyperdivergent Class II cases. Therefore, a larger symphysis allows for a greater chance of a nonextraction approach to treatment. ${ }^{9}$ Conversely, persons with greater symphysis height and a small chin would be candidates for an extraction treatment plan to compensate for arch length discrepancies. ${ }^{9}$ It should be remembered that the symphysis region may limit not only sagittal but also vertical tooth movement, although symphysis height and Id-Id' widths were found to be similar in all groups in the present study. One of the limitations of this study is that the sample distribution was not equal among groups. The hypodivergent malocclusion type had the fewest number of all Class II patients (n=19). This is due to strict patient selection criteria as well. Class II patients with dentoalveolar maxillary protrusions that displayed the hypodivergent facial type were eliminated from this study. As a result, most of the skeletal Class II division 1 patients displayed the hyperdivergent facial type with posterior mandibular rotation. The sample distributions of the present study are similar to those of earlier studies. It may be useful to include larger populations in future studies.

Bone is a dynamic tissue that constantly undergoes remodeling, ${ }^{37}$ but it has been reported that remodeling can remove or conserve the bone but not add to it. ${ }^{38}$ There is disagreement about whether the remodeling capacity of alveolar bone can compensate for bone loss in every case. Some new bone formation at the labial side after incisor retraction would be expected in growing patients but not in nongrowing patients. Furthermore, the probability of encountering tooth loss, bone loss, or periodontal problems increases with age. ${ }^{37,39}$ Gündüz et al ${ }^{37}$ showed new vertical bone formation at the edentulous premolar region with a bodily tooth movement in an adult case, whereas some other authors could not determine new bone formation due to controlled tipping incisor movement..$^{40}$ It is generally 
suggested that rapid tipping tooth movements are to be avoided to minimize the risk of bone dehiscence and root resorption. ${ }^{41}$ In addition to sagittal tooth movements within the lower incisor region, pronounced rotations seem to be critical in cases of narrow alveolar bone support for tooth derotation. ${ }^{16}$

Another point to be considered is that the alveolar bone surrounding the mandibular incisors may not show an equal thickness not realized by the cephalometric films due to superimposition of the anatomic structures. ${ }^{36,42}$ Visualization can be accomplished with high resolution $\mathrm{CT}$, which may be useful for assessing labial and lingual alveolar bone structure and for diagnosing larger dehiscences. However, CT is not yet available for routine orthodontic screening because of financial aspects and additional radiation risks. ${ }^{16}$ Sarıkaya et $a l,{ }^{40}$ in their CT study evaluating alveolar bone thickness after anterior tooth retraction, reported statistically significant decreases in lingual bone width in both arches, and some of their patients demonstrated bone dehiscence that was not visible either macroscopically or cephalometrically. They added that the thickness of the bone lingual to the maxillary lateral incisors decreased more than the thickness of the bone lingual to the centrals. The authors attribute this finding to the teeth having different periodontal ligament areas. Similarly, Gracco et al ${ }^{36}$ found that the total and cancellous heights and areas were greater at the central incisors than at the lateral incisors in all facial types. In another cone beam CT study evaluating the bone surrounding the maxillary incisors irrespective of facial type, the bone thinned progressively from the anterior to the posterior and from the medial to the lateral. ${ }^{43}$ Alveolar bone thickness is very important for determining the suitable location for miniscrews and for determining the limitations of orthodontic tooth movement. These findings emphasize the importance of individual tooth evaluation in both the preand posttreatment periods. In the future, systematic 3D CT analysis of the pre- and postorthodontic findings will contribute to better estimation of the case-specific potential of compensatory bone apposition..$^{41}$

\section{CONCLUSION}

This study was conducted on the lateral cephalograms of 165 patients: 54 (32.7\%) with normal occlusion, $58(35.2 \%)$ with hyperdivergent Class
II division 1, 19 (11.5\%) with hypodivergent Class II division 1, and 34 (20.6\%) with normodivergent Class II division 1. Cervical alveolar width (id -id') were similar in all Class II type. Symphysis width is the main factor in the differential diagnosis of Class II division 1 cases with mandibular retrognathy rather than symphysis height.

Facial type classification has some advantages for diagnosis, prognosis, and treatment planning objectives as well as for distinguishing between dental and skeletal disturbances. The key role of the incisors and the complicated anatomical relationship of this area play a significant role in orthodontic treatment planning.

\section{REFERENCES}

1. Moyers RE., Riolo ML, Guire KE, Wainright RL, Bookstein FL. Differential diagnosis of Class II malocclusion. Am J Orthod 1980;477-494.

2. Henry RG. A classification of Class II, Division I malocclusion. Angle Orthod 1957;27:83-92.

3. Craig CE. The skeletal patterns characteristics of Class I and Class II division I malocclusions in norma lateralis. Angle Orthod 1951;21:44-56.

4. Schudy FF. The rotations of the mandible resulting from growth: it's implications in orthodontic treatment. Angle Orthod 1965;35:36-53.

5. Sassouni V. The Class II syndrome: Differential diagnosis and treatment. Angle Orthod 1970;40:334-341.

6. Hellman M. Studies on the etiology of Angle's class II malocclusion manifestations. Internat $J$ Orthodontics 1922;8:129-159.

7. Drelich RC. A cephalometric study of untreated Class II Division 1 malocclusion. Angle Orthod 1948;18:70-75.

8. Bishara SE, Augspurger EF. The role of mandibuler plane angle inclination in orthodontic diagnosis. Angle Orthod 1975;45:273-281.

9. Aki T, Nanda RS, Currier GF, Nanda SK. Assessment of the symphysis morphology as a predictor of the direction of mandibular growth. Am J Orthod 1994;106:60-69.

10. Bjork A. Prediction of mandibular growth rotation. Am J Orthod 1969;55:585 599.

11. Isaacson JR, Isaacson RJ, Speidel TM, Worms FW. Extreme variation in vertical facial growth and associated variation in skeletal and dental relations. Angle Orthod 1971;41:219229.

12. Buschang PH, Julien K, Sachdeva R, Demirjian A. Childhood and pubertal growth changes of the human symphysis. Angle Orthod 1992;62:203-210. 
13. Enlow DH, Harris DB. A study of the postnatal growth of the human mandible. Am J Orthod 1964;75:25-50.

14. Skieller V, Bjork A, Linde-Hansen T. Prediction of mandibular growth rotation evaluated from a longitudinal sample. Am J Orthod 1984;86:359-370.

15. Karlsen AT. Craniofacial growth differences between low and high MP-SN angle males: a longitudinal study. Angle Orthod 1995;65:341-350.

16. Wehrbein H, Bauer W, Diedrich P. Mandibular incisors, alveolar bone, and symphysis after orthodontic treatment. A retrospective study. Am J Orthod 1996;110:239-246.

17. Edwards JG. A study of the anterior portion of the palate as it relates to orthodontic therapy. Am J Orthod 1976;69:249273.

18. Kim YS, Cha JY, Yu HS, Hwang CJ. Comparison of mandibular anterior alveolar bone thickness in different facial skeletal types. Korean J Orthod 2010;40:314-324.

19. Tweed $\mathrm{CH}$. The Frankfort mandibular plane angle in orthodontic diagnosis, classification, treatment planning and prognosis. Am J Orthod 1946;32:175-230.

20. Kim YK, Kyung HM, Kwon OW, Sung JH. Roentgenographic cephalometric study of angle's class II, division 1 malocclusion in Korean children. Korean J Orthod 1989;19:67-78.

21. Jeon LO, Lee KS. The cephalometric study of facial types in Class II division 1 malocclusion. Korean J Orthod 1989;19:201-218.

22. Altemus LA. Horizontal and vertical dentofacial relationship in normal and Class II division 1 malocclusion in girls 11-15 years. Angle Orthod 1955;25:120-137.

23. Gilmore WA. Morphology of the adult mandible in Class II Division 1 malocclusion and in excellent occlusion. Angle Orthod 1950;20:137-146.

24. Blair ES. A cephalometric roentgenographic appraisal of the skeletal morphology of Class I, Class II Div 1 and Class II Div 2 (Angle) malocclusions. Angle Orthod 1954;24:106119.

25. Downs WB. Variation in Facial relationships: their significance in treatment and prognosis. Am J Orthod 1948;34:812840.

26. Margolis HI. The axial inclination of the mandibular incisors. Am J Orthod 1943;29:571.

27. Opdebeek H, Bell WH, Eisenfeld J, Mishelevich D. Comparative study between the SFS and LFS rotation as a possible morphogenetic mechanism. Am J Orthod 1978;74:509-521.

28. Fields HW, Proffit WR, Nixon WL, Phillips C, Stanek E. Facial pattern differences in long-faced children and adults. Am J Orthod 1984;85:217-223.

29. Baumrind S, Bravo LA, Ben-Bassat Y, Curry S, Korn EL. Lower molar and incisor displacement associated with mandibular remodeling. Angle Orthod 1997;67:93-102.
30. Janson GRP, Metaxas A, Woodside DG. Variation in maxillary and mandibular molar incisor vertical dimension in 12-year old subjects with excess, normal and short lower anterior face height. Am J Orthod 1994;106:409-418.

31. Rosenstein SW. A longitudinal study of anteroposterior growth of the mandibular symphysis. Angle Orthod 1964;34:155-167.

32. Garn SM, Lewis AB, Vicinus JH. The inheritance of symphyseal size during growth. 1963;33:222-231.

33. Noh SH, Lee KS, Park YK. A cephalometric study on correlation between mandibular symphysis and craniofacial skeleton. Korean J Orthod 1997;27:119-127.

34. Kim SJ, Son WS. A study on the relationship of the mandibular symphysis and anterior alveolar and skeletal morphology according to the rotational growth pattern of mandible in skeletal Class III malocclusion Korean J Orthod 1999;29:303-315.

35. Tang N, Zhao ZH, Liao CH, Zhao MY. Morphological characteristics of mandibular symphysis in adult skeletal class II and class III malocclusions with abnormal vertical skeletal patterns. Hua Xi Kou Qiang Yi Xue Za Zhi 2010;28:395-398.

36. Gracco A, Luca L, Bongiorno BC, Siciliani G. Computed tomography evaluation of mandibular incisor bony support in untreated patients. Am J Orthod Dentofac Orthop 2010;138:179-187.

37. Gündüz E, Rodriguez-Torres C, Gahleitner A, Heissenberger G, Bantleon HP. Bone regeneration by bodily tooth movement: dental computed tomography examination of a patient. Am J Orthod 2004;125:100-106.

38. Frost HM. Walff's law and bone structural adaptations to mechanical usage: an overview for clinicians. Angle Orthod 1994;3:175-188.

39. Lupi JE, Handelman CS, Sadowsky C. Prevalence and severity of apical root resorption and alveolar bone loss in orthodontically treated adults. Am J Orthod Dentofacial Orthop 1996;109:28-37.

40. Sarıkaya S, Haydar B, Ciğer S, Arıyürek M. Changes in alveolar bone thickness due to retraction of anterior teeth. Am J Orthod 2002;122:15-26.

41. Diedrich P. Problems and risks in the movement of the mandibular anterior teeth. Fortschr Kieferorthop 1995;56:148156.

42. Yamada C, Kitai N, Kakimoto N, Murakami S, Furukawa S, Takada K. Spatial relationships between the mandibular central incisor and associated alveolar bone in adults with mandibular prognathism. Angle Orthod 2007;77:766-772.

43. Gracco A, Luca L, Cozzani M, Siciliani G. Assessment of palatal bone thickness in adults with cone beam computerised tomography. Aust Orthod J 2007:23;109-113. 